\section{Mounting Media and Antifade Reagents}

\author{
Compiled ${ }^{1}$ by Tony J. Collins, \\ Wright Cell Imaging Facility, Toronto Western Research Institute \\ tonyc@uhnresearch.ca
}

In the biomedical sciences, samples are mounted in a wide variety of media for examination by microscope. There are a wide variety of mounting media available with a correspondingly wide range of properties. Using the incorrect mounting medium may cause signal loss and optical aberrations; the correct mounting medium avoids such aberrations and preserves fluorescence signal with "anti-fading" properties. This article introduces mounting media for fluorescence microscopy, providing descriptions of their constituents and their properties, as well as accounts of users' experience

More detailed reviews of antifade reagents have been published by Ono et al. (1) and Longin et al.(2). Papers describing the effect of refractive index (RI) mismatch have been published by Diaspro et al. (3) and Hell et al. (4).

\section{Refractive index mismatch}

Light is refracted when it crosses the interface between two media of differing refractive indices (RI) (Fig 1). Mismatching the refractive index of the objective immersion medium ${ }^{2}$ and the mounting medium is one of the main causes of image degradation in microscopy. Refractive index mismatch results in stretching/compression of the $\mathrm{z}$-axis: a $1 \mu \mathrm{m}$ step size for the objective does not necessarily correspond to a $1 \mu \mathrm{m}$ change in the focal plane. Also, spherical aberration is worsened by axial spreading of the point-spread function (PSF) resulting in reduced axial resolution (3). Another consequence of the axial spreading of the PSF is signal-loss in a confocal system since fewer photons pass through the pinhole (J. Pawley, 1999, CLA). This phenomenon is exacerbated with depth; with a high numerical aperture objective, serious problems arise when imaging deeper than $10 \mu \mathrm{m}$ into an aqueous sample (5). The mounting medium and the immersion medium should be matched within 0.01-0.05 (B. Foster, 2003, CLA) ideally to three decimal places (J. Pawley, 1997, CLA).

It should also be noted that the refractive index of an immersion medium will vary with temperature approximately 0.0004 per $1^{\circ} \mathrm{C}$ (J. Cargille, Cargille Labs); the variance will be even more pronounced with wavelength.

\section{Constituents}

There are many commercial mounting media available, and even more recipes to make your own. Mounting media are

1 This information is a collation of useful comments from the Confocal listserver archives (CLA) and the Histonet archives (HA) as well as other web-resources. The original comments can be found by searching for the author and year in the CLA archive or in the Histonet archive by selecting the appropriate archive (defined by the month and date) and searching for the author's name in the page. A PDF version of this article is available from the Documents section of the Wright Cell Imaging Facility website with clickable links to the archived comments. It is the readers responsibility to take all appropriate safety precautions when using any of the formulations or protocols mentioned in this article and to adhere to all local, state and federal regulations that may apply. Also see the Disclaimer Notice on page 6 of this issue. Neither the author, the author's employer, Microscopy Today and its staff, or the Microscopy Society of America can accept any legal responsibility with regard to your use of the material in this article. The opinions expressed in this article on the relative merits of any firm, product, or service mentioned herein are solely those of the author.

2 In this article "immersion medium" refers to the medium between the objective and coverslip, i.e. the "immersion medium" for air-objectives is air.

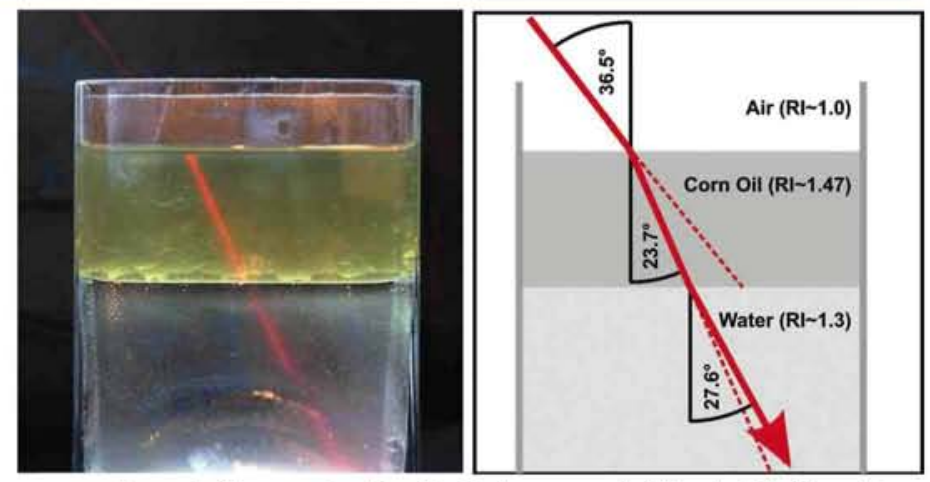

Figure 1: Changes in refractive index causes light to deviate from its expected path. A laser-pointer is shone through air into a vase containing corn oil on top of water. A little talc is included in the air, oil, and water

in order to improve visualization by scattering the laser-light.

typically comprised of two or three main ingredients: a base, an antifade reagent, and sometimes a plasticizer to set.

\section{Base constituents}

The base component of a mounting medium is either aqueous (RI 1.33); glycerol (RI 1.47); natural oil (RI 1.53); or plastic (RI 1.51). The base-ingredient is the major determinate of the refractive index of the medium (W. Wallace, 2001, CLA). Natural oils and plastic-base mounting media tend to be hydrophobic, and therefore require dehydration of the sample: this may cause significant shrinkage of tissue (J. Pawley, 2003, CLA). Aqueous and glycerol-based media are hydrophilic and do not require the sample to be dehydrated.

Glycerol may be incompatible with specimens containing lipophilic plasma membrane stains like DiI; this may leach (P. Jansma, 1999, CLA; W. Wallace, 2002, CLA). However, it should be noted that others have not had this problem (A. Elberger, 1999, CLA).

\section{Antifade constituents}

The mechanism by which fluorescent dyes are irreversibly destroyed by photobleaching is not fully understood. It is thought to be linked to the transition from the excited singlet state to the excited triplet state (the latter is more reactive). In this triplet state, the dyes may react with molecular oxygen and lose the ability to become fluorescent, i.e. photobleached. However, photobleaching has been demonstrated in an argon environment, in the absence of oxygen (6). This may suggest triplet dye-dye interactions (7), which may be strongly dependent on dye concentration and dye saturation state (K. Braeckmans, 2003, CLA). Most antifade reagents are reactive oxygen species scavengers. However, sometimes the antifade itself will quench the fluorescence of the dye but prolong its life at this intensity (K.Larison, Apr 1999, HA).

p-Phenylenediamine (PPD) seems to be the most effective antifade compound (1)(6). However, it can react with cyanine dyes (especially $\mathrm{Cy} 2$ ) and cleave away half of the cyanine molecule (Jackson ImmunoResearch Laboratories, TechFAQ\#3). Use of phenylenediamine as an anti-fading reagent in mounting media may result in weak and diffused fluorescence following storage of the stained slides. There are several reports of ultraviolet-induced yellow-fluorescence of Hoechst-stained nuclei. One possible explanation of this phenomenon is the contamination of the mounting medium with meta-phenylenediamine. This may react in the presence of weak acid to form a yellow-fluorescing basophilic stain. Avoid anything that might be a mixture of the 


\section{Explore, \\ Discover, Build.}

\section{$\because$ FEI COMPANY \\ TOOLS FOR NANOTECH}

Today's advanced discovery and product development requires

unimpeded 3D access to the nanoscale with the best resolution and accuracy possible. FEI's Tools for Nanotech ${ }^{\mathrm{TM}}$ support the success of your most demanding projects by enabling you to reach far into the nanoscale and obtain the complete data you need.

From introducing the first DualBeam ${ }^{\mathrm{TM}}$ systems more than 10 years ago, to being the first to break the Ångström barrier on a commercially-available TEM, FEl's leadership continues to deliver essential tools and advanced applications.

In the race for nanoscale discovery and commercialization you can't wait until tomorrow for the tools you need today. You can always rely on FEl, the world's leader in Tools for Nanotech, to give you more access to the nanoscale so you can explore, discover and build like you've never imagined.

\section{With Unparalleled Access to the Nanoscale}


Table1: Refractive Indices. A more comprehensive list has been compiled by George McNamara (2003, CLA). Variation may be due to differences in the wavelength and temperature used to measure R.I.

\begin{tabular}{|c|c|}
\hline Medium & Refractive Index \\
\hline Vacuum & 1.00 , by definition \\
\hline Air & $\sim 1.00$ \\
\hline Water & 1.333 \\
\hline Cells & $\sim 1.43$ (A. Diaspro, 2000, CLA) \\
\hline Glycerol & $\begin{array}{c}1.45 \text { (G. Macnamara, 2003, CLA) }-1.466 \\
\text { (Microscopia Optica y Confocal) }-1.48(3)\end{array}$ \\
\hline $\begin{array}{c}\text { Borosilicate glass } \\
\text { (e.g. Pyrex") }\end{array}$ & 1.476 (Melles Griot) \\
\hline Optical Crown glass & 1.517 (Olympus) -1.52501 (Melles Griot) \\
\hline Fixed protein & 1.54 (R. Ellis) \\
\hline DIN std. immersion oil 1 & 2.419 \\
\hline Diamond & 3.5 \\
\hline Gallium phosphide & 0.0004 (DIN), +1-0.0005 (ISO) \\
\hline
\end{tabular}

The DIN (Deutsches Institute fur Normung) standard for measuring refractive index of immersion oil is specified at one wavelength, generally the "mercury e" line $(546.1 \mathrm{~nm})$ at $23^{\circ} \mathrm{C}$ (Cargille Labs). It is predicted that ISO standards being drafted will be based on the DIN standard (Cargille Labs).

para- and meta-isomers (M. Wessendorf, 1993, CLA).

$\boldsymbol{n}$-Propyl gallate (NPG) is another commonly used antifade compound. It is difficult to dissolve, requiring prolonged heating over several hours. It is non-toxic and can be used with live cells. However, it has been shown to protect against apoptosis (8), so it may interfere with the biological process under study.

1,4-Diazabicyclo-octane (DABCO), also known as triethylenediamine, is not as effective as PPD (1) but is less toxic. It could be used in live-cell work; however, it would be predicted to have the same anti-apoptotic properties of NPG.

\section{To Harden or not to Harden?}

Some mounting media harden as they dry. This facilitates handling and storage; however, the process of hardening often results in significant changes in the RI over time. During the hardening process, there can also be shrinkage and tissue damage. However, this shrinkage may be insignificant compared to the shrinkage that results from dehydration during the fixation process (J. Pawley, 2003, CLA).

There are mounting media which do not harden. These require sealing the coverslip in order to prevent the mounting medium drying or leaking. Spacers may be needed to prevent the coverslip from crushing the specimen.

\section{Sealants}

Nail polish is widely used to seal the edges of coverslips. There are many accounts of nail polish quenching GFP (J. Feijo, 2002, CLA; S. Castel, 2002, CLA) but others have not had a problem (M. Mancini, 2002, CLA; A. Cornea, 2002, CLA). The quenching is possibly linked to the water-soluble isopropyl alcohol in some nail polishes (G. Callis, 2003, CLA).

Allow at least 30 minutes for nail polish to dry. Sealants that have not been thoroughly dried will damage the lenses. If you do get polish on the objective lens it may be chipped off with a toothpick - the wood in toothpicks is reported to be soft enough not to damage the lens, although it is recommended to check with the objective manufacturer before attempting this (M. Wessendorf, 2001, CLA).

Entellan is sold as a xylene-based mounting medium like DPX (see below) but contains poly (methyl methacrylate) rather than polystyrene (80kD) (J. Kiernan, May 2000, HA). Although sold as a mounting medium, Entellan has been recommended as a sealant as it sets quickly (W. Wallace, 2002, CLA) (20 minutes) and the best sealant for glycerol-based mounting media (W. Wallace, 2001, CLA).

Dental or modelling wax comes as pink coloured sheets and can be safely used and easily reversed. Heat the sheet in a glass beaker and it will become liquid at $40^{\circ} \mathrm{C}-60^{\circ} \mathrm{C}$. Use a small paintbrush for sealing the coverslip. When the wax is in touch with the coverslips at room temperature it will solidify (S. Castel, 2004, CLA).

VALAP is an equal mixture of Vaseline, lanolin, and paraffin mixed on a heating plate. VALAP can be used to seal living samples: its low melting point prevents damaging the sample when applied (M. Farmer, 2004, CLA).

\section{Spacers}

Broken coverslips have been recommended as inexpensive permanent spacers $(\# 1=0.13-0.17 \mathrm{~mm} ; \# 1.5=0.16-0.19 \mathrm{~mm}$; $\# 2=0.17-0.25 \mathrm{~mm}$ thick). These can be precisely cut with a diamond-pen (G. Macdonald, 1995, CLA).

Gaskets are commercially available (or can be made from latex gloves (M. Montrose, 1994, CLA) or Parafilm (G. Macdonald, 1995, CLA)). Molecular Probes supply silicone gaskets ranging from $0.2 \mathrm{~mm}$ to $2.5 \mathrm{~mm}$.

Scotch tape has also been reported to work as a $60-100 \mu \mathrm{m}$ spacer (C. Montrose, 1999, CLA), but may be autofluorescent (G. Macdonald, 1995, CLA).

Custom-made metal spacers have been found to be useful in live cell work where they can be re-used (B. Foster, 1998, CLA; R. Thrift, 1995, CLA).

\section{Commercially Available Mounting media}

The following summary of commercially available mounting media has been taken from comments made on the confocal listserver, IHC archive, and Microscopy websites around the world. There is less material on new products, which have yet to be fully evaluated.

\section{Aqueous}

Gel Mount is an aqueous-based mounting. The manufacturers say that it has been specially formulated without glycerol in order to avoid the deleterious effects it has in some of the phycobiliprotein-based detection systems (there is some evidence that glycerol can change the fluorescence decay properties of phycobiliproteins (9)). Gel/Mount does not contain phenylenediamine, which may interfere with cyanine dyes.

FluorSave has been highly recommended. It hardens after about 1 hour, has strong antifade properties, but as with other hard-setting mounting media, shrinkage after 1 week can damage tissue (W. Wallace, Feb 2002, CLA).

\section{Glycerol-based}

Prolong is a two-component mounting medium, which begins as an antifade powder and a liquid-base component. The 
two components are mixed just prior to use. This 'T product has recently been joined by Prolong Gold (which comes premixed but is more expensive per $\mathrm{ml}$.). Prolong is reported not to quench fluorescence like some other antifades (K. Larison, Apr 1999, HA). While it is said to be good for the AlexaFluor dyes, it may not work so well with fluorescent proteins (K. Larison, Apr 1999, HA), although Clontech recommends it (Clontech, Living Colors FAQ: Fixation of Fluorescent Proteins). It is reported that Prolong stores better than Vectashield and probably uses PPD as an antifade (T. Howard, 1999, CLA). Molecular Probes report that Prolong does not work well with BODIPY dyes.

Vectashield comes as regular or hard-set. It has been suggested that it does not work well with Cyanine dyes (possibly because its antifade reagent is PPD). Vectashield suffers from a blue autofluorescence when excited with UV; this, however, fades quickly. Autofluorescence may be exacerbated by storage at $-20^{\circ} \mathrm{C}$ ( T. Howard, 1999 , CLA). Vectorlabs state that Vectashield may not be compatible with all enzymatic substrates or fluorescent proteins.

MOWIOL is a PVA-based medium originally designed for EM. The refractive index is inconsistent between batches (W. Wallace, Feb 2002, CLA), possibly due to the fact that it arrives as a powder and requires reconstitution in glycerol and buffer (see Appendix for recipes).

\section{Plastic}

$D P X$ is mixture of distyrene (a polystyrene), a plasticiser (tricresyl phosphate), and xylene (DPX). This compound was introduced in 1939 and later modified by the substitution of a more satisfactory plasticiser, dibutylphthalate (butyl, phthalate, styrene - BPS). Given the presence of xylene in this compound, procedures must be performed in a fume hood using forceps.

DPX requires tissue dehydration (this process compresses structures in the z-axis, and will extract any lipid-soluble fluorophores).

DPX is reported to work well with TRITC, Lissamine rhodamine, rhodamine Red-X, Cy2, Cy3, or Cy5; however it appears to be less successful with FITC or AMCLA (M. Wessendorf, 1999, CLA).

Cytoseal 60 is an inexpensive, toluene-based low viscosity mounting medium (E. Monosov, 2000, CLA). As with Entellan, its base constituent is poly(methylmethacrylate). It can form bubbles when drying (J. Kiernan, Mar 2005, HA) and is considered inferior to the similar Entellan (J. Kiernan, Mar 2003A, HA).

Permount is a toluene-based synthetic resin mounting medium. The product sets more quickly than DPX and has a lower viscosity.

\section{Web resources}

This article is available at Wright Cell Imaging Facility website with live in-text links to the original articles: http://www. uhnresearch.ca/wcif/download.html

Confocal listserver archives: http://listserv.buffalo.edu

(1)

${ }^{1}$ From Confocal list, supplier's websites and Servicio de Microscopia Óptica y Confocal:http://www2. cbm.uam.es/confocal/Ingles/default_english.htm

${ }^{4}$ One unit is $2.4 \mathrm{~g}$ which is dissolved in $24 \mathrm{ml}$.

Histonet archives: http://www.histosearch.com/histonet. html

Microscopía Optica y Confocal Refractive Index table for a number of mounting and immersion media http://www2.cbm. uam.es/confocal/Manuales/Indices\%20refraccion\%20web. pdf

\section{Recipes}

There are many recipes available to make your own mounting medium. These may be cheaper than commercial products but also more variable in their refractive indices. Most of these are glycerol-based mounting media. There are reports that some batches/sources of glycerol can autofluoresce; check new bottles before preparing the mounting media (T. Howard, 2003, CLA). PPD-Glycerol I

The key to using para-phenylenediamine is $\mathrm{pH}$ : if the $\mathrm{pH}$ is below $\sim 8.0$, you will see fading and background signal. Bicarbonate is used here to maintain the $\mathrm{pH}-$ level.

$0.1-0.01 \%$ p-phenylenediamine

$50-70 \%$ glycerol in PBS50\% glycerol in $20 \mathrm{mM}$ phosphate buffer ( $\mathrm{pH} 8.5)$

Aliquot and store at $-80^{\circ} \mathrm{C}$. The medium will go dark when it goes off (E. Hines, 1993, CLA).

\section{PPD-Glycerol II (6)}

$1 \mathrm{~g} / \mathrm{l}(10 \mathrm{mM})$ PPD $90 \%$ glycerol in PBS pH 8.6 adjusted with $0.1 \mathrm{M} \mathrm{NaOH}$ 
PPD dissolved in PBS before addition of glycerol.

DABCO-PVA

$2.5 \%$ DABCO

$10 \%$ polyvinyl alcohol (PVA) (Sigma; Type II),

$5 \%$ glycerol,

$25 \mathrm{mM}$ Tris buffer, $\mathrm{pH} 8.7$

Day 1

1. Add $4.8 \mathrm{~g}$ of polyvinyl alcohol (PVA) to $12 \mathrm{~g}$ of glycerol and mix well.

2. Add $12 \mathrm{ml}$ of distilled water and leave it on a rotator at room temperature overnight.

Day 2

3. Add $24 \mathrm{ml}$ of $0.2 \mathrm{M}$ Tris- $\mathrm{HCl}$ at $\mathrm{pH} 8-8.5$.

4. Heat in a water bath to $50^{\circ} \mathrm{C}$ while mixing for about 30 minutes.

5. Add $1.25 \mathrm{~g}$ of DABCO and mix well.

6. Centrifuge at about $2000 \mathrm{rpm}$ for 5 minutes.

7. Aliquot the supernatant (we use $1 \mathrm{ml}$ aliquots, which is enough for $15-20$ slides) and store at $-20^{\circ} \mathrm{C}$.

Since it polymerizes upon contact with air it is better not to refreeze the aliquots. Thaw aliquot just before use and throw away what you do not use (J. Wassélius, 1999, CLA).

\section{NPG-Glycerol}

$2 \% \mathrm{n}$-propyl-gallate (Sigma)

$49 \%$ PBS

$49 \%$ glycerol

$\mathrm{pH}=7.4$.

\section{DABCO-Glycerol}

$1 \%$ DABCO in $90 \%$ glycerol, phosphate buffered (6).

Dissolve DABCO in glycerol with gentle heating before addition to PBS. Samples seem to last for weeks to years if kept in the dark (G. Macdonald, 1996, CLA)

\section{MOWIOL-DABCO}

Add 2.4g of MOWIOL 4-88 to $6 \mathrm{~g}$ of glycerol. Stir to mix.

Add $6 \mathrm{ml}$ of water, leave stirring at room temperature for several hours

Add $12 \mathrm{ml}$ of $0.2 \mathrm{M}$ Tris [pH8.5] and heat to $50 \mathrm{C}$ for $10 \mathrm{~min}$ with occasional mixing.

Clarify by centrifugation at $5,000 \mathrm{~g}$ for 15 minutes.

OPTIONAL: Add DABCO [1,4,-diazobicycli-[2.2.2]-octane, Aldrich] to $2.5 \% \mathrm{w} / \mathrm{v}$ to reduce fading of fluorophores.

RT (C. Favara, 2003, CLA).

Aliquot in airtight tubes and store at $-20^{\circ} \mathrm{C}$. Stocks are stable at $4^{\circ} \mathrm{C}$ for several weeks after thawing (J. Trogadis, 2003, CLA)].

\section{Reference List}

(1) Ono et al. J Histochem Cytochem 2001; 49(3):305-312.

(2) Longin et al. J Histochem Cytochem 1993; 41(12):1833-1840.

(3) Diaspro et al. Appl Opt 2002; 41(4):685-690.

(4) Hell et al. Journal of Microscopy 1993; 169(3):391-105.

(5) Bacallao et al. In: Pawley JB, editor. Handbook of confocal icroscopy. New York: Plenum Press, 1995: p311-325.

(6) Johnson et al. Journal of Immunological Methods 1982; 55(2):231-242.

(7) Song L et al. Biophys J 1995; 68(6):2588-2600.

(8) Paz-Miguel et al. J Immunol 1999; 163(10):5399-5410.

(9) Steglich et al. FEBS Letters 2003; 553(1-2):79-84.

\section{NWRODUCING
The New High-Resolution Turbo Sputker Coater}

the range
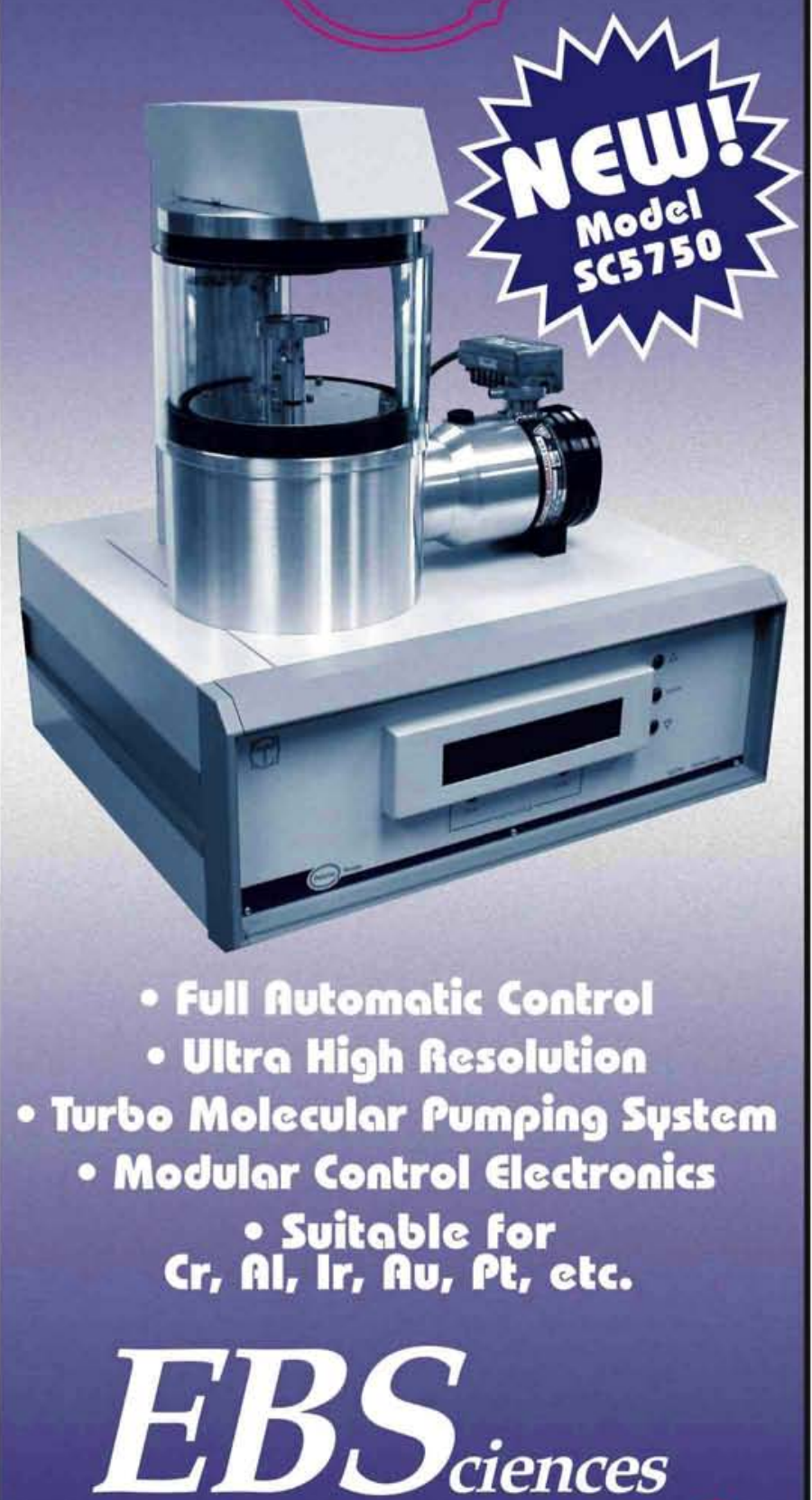

ADDING BRILLIANCE TO YOUR VISION

800-992-9037 or 860-635-0411

e-mail: ebs@ebsciences.com www.ebsciences.com 